\title{
Treating acute large vessel occlusion stroke: to bridge or not to bridge?
}

\author{
Yunyun Xiong (D) ,, ${ }^{1,2}$ Yuesong Pan (D) , ${ }^{2}$ Raul G Nogueira, ${ }^{3}$ Zeguang Ren (D) , \\ Tudor G Jovin, ${ }^{5,6}$ Yongjun Wang (D) ${ }^{2}$
}

To cite: Xiong Y, Pan Y, Nogueira RG, et al. Treating acute large vessel occlusion stroke: to bridge or not to bridge?. Stroke \& Vascular Neurology 2021;6: e000952. doi:10.1136/svn-2021-000952

Received 18 February 2021 Revised 2 March 2021 Accepted 3 March 2021 Published Online First 26 April 2021
Check for updates

(c) Author(s) (or their employer(s)) 2021. Re-use permitted under CC BY-NC. No commercial re-use. See rights and permissions. Published by BMJ.

${ }^{1}$ Neurology, Beijing Tiantan Hospital, Capital Medical University, Beijing, China ${ }^{2}$ China National Clinical Research Center for

Neurological Diseases, Beijing Tiantan Hospital, Capital Medical University, Beijing, China

${ }^{3}$ Neurology, Marcus Stroke and Neuroscience Center, Grady Memorial Hospital, Emory University School of Medicine, Atlanta, Georgia, USA

${ }^{4}$ Neurosurgery, Cleveland Clinic Martin Health, Port St Lucie, Florida, USA

${ }^{5}$ Cooper Neurologic Institute, Cooper University Hospital, Camden, New Jersey, USA

${ }^{6}$ Neurology, Cooper Medical School of Rowan University, Camden, New Jersey, USA

Correspondence to Dr Yongjun Wang; yongjunwang@ncrcnd.org.cn
Hot debates exist regarding whether patients who had an acute large vessel occlusion stroke (LVOS) can skip tissue plasminogen activator (tPA) and go directly to thrombectomy in comprehensive stroke centres. Three headto-head randomised clinical trials (RCTs) of direct endovascular treatment (dEVT) versus bridge therapy (BT) with intravenous alteplase have been recently completed in the Asian population. The DIRECT-MT (Direct Intraarterial Thrombectomy in Order to Revascularize Acute Ischemic Stroke Patients with Large Vessel Occlusion Efficiently in Chinese Tertiary Hospitals: a Multicenter Randomized Clinical Trial) Study randomly enrolled 656 patients who had an acute ischaemic stroke with anterior circulation LVOS across 41 large teaching hospitals in China to receive BT or dEVT within 4.5 hours after symptom onset. ${ }^{1}$ The study showed that the dEVT group was non-inferior to BT in terms of 90-day clinical outcomes (median 90-day modified Rankin Scale (mRS), 3 vs 3; OR $1.07 ; 95 \%$ CI 0.81 to $1.40 ; \mathrm{p}=0.04) .{ }^{1}$ Likewise, the recently published DEVT (Direct Endovascular Thrombectomy vs Combined IVT and Endovascular Thrombectomy for Patients With Acute Large Vessel Occlusion in the Anterior Circulation) trial involving 234 Chinese patients who had an anterior circulation LVOS found that the rate of functional independence (90-day $\mathrm{mRS} \geq 2$ ) in dEVT group was numerically higher than that in the BT group $(54.3 \%$ vs $46.6 \%$, difference: $7.7 \%$, 1-sided $97.5 \%$ CI: $-5.1 \%$ to $\infty ; \mathrm{p}=0.003$ for non-inferiority). ${ }^{2}$ With prespecified noninferiority threshold of $10 \%$ for the favourable outcome proportion difference, the trial concluded that dEVT was non-inferior to BT. ${ }^{2}$ Simultaneously published with DEVT, the SKIP (Direct Mechanical Thrombectomy in Acute LVO Stroke) trial included 204 patients who had an acute ischaemic stroke with internal carotid artery or middle cerebral artery M1 occlusions presenting within 4.5 hours of stroke onset across 23 sites in Japan. ${ }^{3}$ Favourable outcome occurred in 60 patients
$(59.4 \%)$ in the dEVT group and 59 patients $(57.3 \%)$ in the BT group, with no significant between-group difference (difference, $2.1 \%$ (1-sided $97.5 \% \mathrm{CI}-11.4 \%$ to $\infty$ ); OR 1.09 (1-sided $97.5 \%$ CI 0.63 to $\infty$ ); $p=0.18$ for non-inferiority). ${ }^{3}$ Noted that the trial used a reduced dose of alteplase $(0.6 \mathrm{mg} / \mathrm{kg})$, which did not show the non-inferiority to standard dose of alteplase $(0.9 \mathrm{mg} / \mathrm{kg}){ }^{4}$

Herein, we provide an aggregate data metaanalysis of the DIRECT-MT, ${ }^{1}$ the DEVT ${ }^{2}$ and the SKIP $^{3}$ trials. The OR for 90-day functional independence (90-day mRS 0-2) was 1.08 (95\% CI 0.85 to 1.38 ) while the OR for any 1-point improvement across the disability scale (ordinal shift 90-day mRS) was 1.06 (95\% CI 0.86 to 1.31$)$. The percentage of achieving a Thrombolysis in Cerebral Infarction score $2 b-3$ was numerically higher in the bridging group, around $5 \%$ in DIRECT-MT ${ }^{1}$ and $3 \%$ in SKIP. ${ }^{3}$ The rates of successful recanalisation, 90-day mortality, and symptomatic intracerebral haemorrhage were not significantly different between the dEVT and the BT groups (figure 1).

\section{MERITS AND SHORTCOMINGS}

These trials were well-conducted head-to-head comparisons of dEVT with BT in tPA-eligible patients. There was no significant delay of door-to-puncture time between dEVT and BT groups across these trials, which facilitated the direct comparisons between these two strategies. However, there are some aspects related to the study design that deserve additional discussions when considering the clinical implications of these three trials.

A major concern for the direct endovascular thrombectomy is the generous boundary for non-inferiority margins of the three randomised trials. ${ }^{5}$ The DIRECT-MT ${ }^{1}$ set a 0.80 for OR, the $\mathrm{SKIP}^{3}$ set a 0.74 for OR as the non-inferiority margin, whereas the DEVT ${ }^{2}$ set a $10 \%$ absolute difference of proportion of favourable functional outcome as the noninferiority margin. All the non-inferiority 


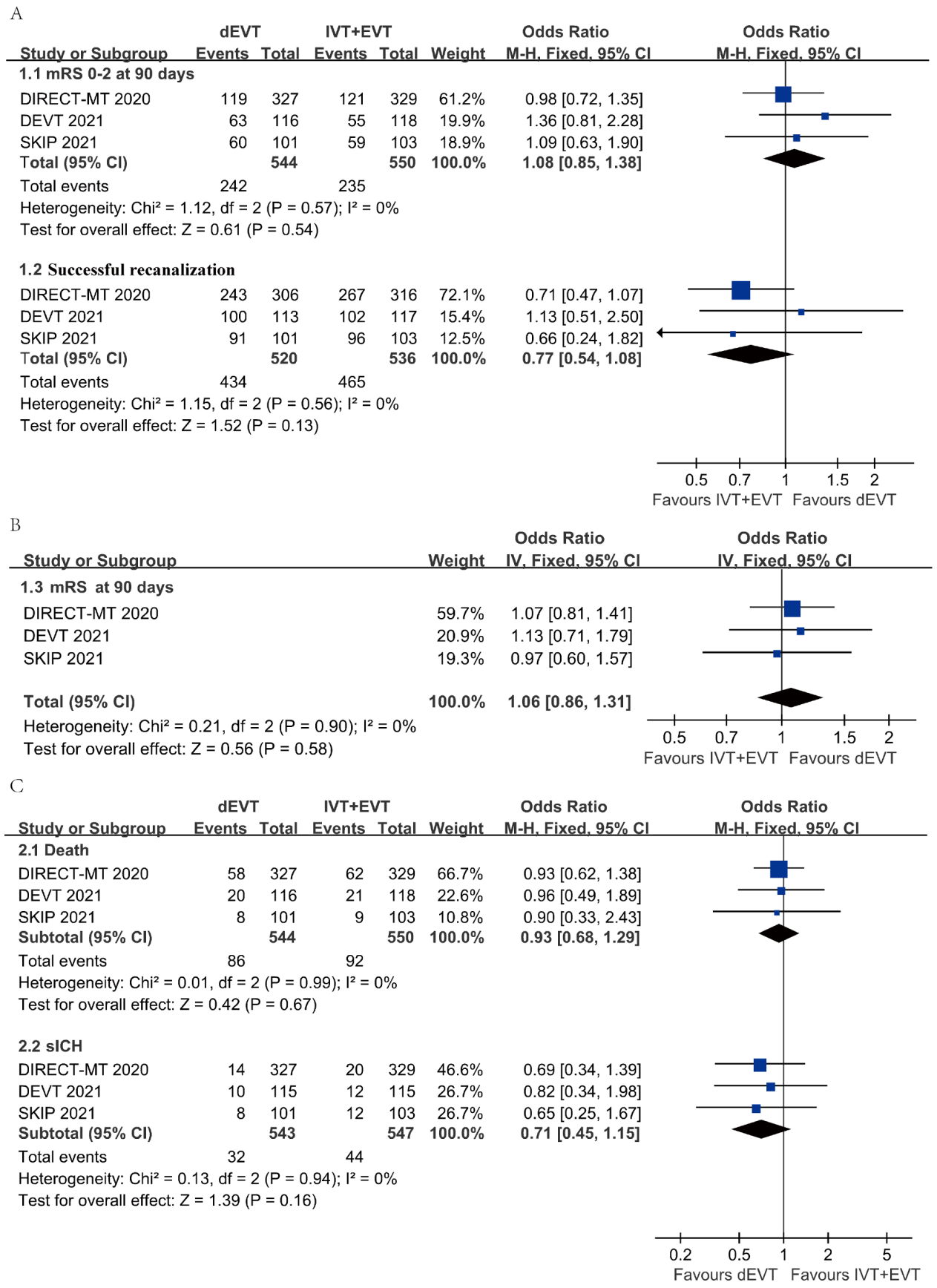

Figure 1 Meta-analysis of the efficacy and safety outcomes of three randomised trials. (A) $1.1 \mathrm{mRS} 0-2 ; 1.2$ successful recanalisation (eTICI $\geq 2 \mathrm{~b}$ in the DIRECT-MT ${ }^{1}$ and DEVT, ${ }^{2}$ and $\mathrm{TICl} \geq 2 \mathrm{~b}$ in the SKIP ${ }^{3}$; (B) $1.3 \mathrm{mRS}$ at 90 days; (C) 2.1 death; 2.2 sICH (Heidelberg criteria in the DIRECT-MT ${ }^{1}$; NINDS criteria in the DEVT ${ }^{2}$ and the SKIP. ${ }^{3}$ dEVT, direct EVT; eTICI, expanded Thrombolysis in Cerebral Infarction; EVT, endovascular thrombectomy; IVT, intravenous thrombolysis; mRS, modified Rankin Scale; NINDS, National Institute of Neurological Disorders and Stroke; sICH, symptomatic intracerebral haemorrhage.

margins used in the three trials were clearly lower than the minimal clinically important differences (MCIDs) recommended by stroke expert survey studies, which suggested MCIDs of $3.5 \%-4.4 \%$ proportion of the functional independence and a stricter criterion of $1 \%-1.5 \%$ in novel endovascular thrombectomy devices trials for acute ischaemic stroke. ${ }^{6}$ Even lower boundary of CI of the combined OR based on the meta-analysis of the three randomised trials (figure 1) only marginally reached the suggested non-inferiority margin of OR 0.85 (corresponding to $4 \%$ proportion of the functional independence suggested by stroke expert survey studies, median of $3.5 \%$ and $4.4 \%$ ). Therefore, the unequivocal non-inferiority of direct versus bridging endovascular thrombectomy remains controversial and requires further validation.

The door-to-needle times of DIRECT-MT, ${ }^{1}$ DEVT $^{2}$ and SKIP $^{3}$ were 59 (45-78) min, 61 (49-81) min and $50 \mathrm{~min}$, respectively, which represent a substantial delay in relation to the 40 (29-55) min reported in the HERMES meta-analysis (Highly Effective Reperfusion Evaluated 
in Multiple Endovascular Stroke Trials) and may have resulted in lower rates of favourable outcomes than what would have been accomplished with shorter door-toneedle times. ${ }^{7}$ Moreover, the rates of favourable outcomes noted in DIRECT-MT ${ }^{1}$ were significantly lower than those noted in DEVT ${ }^{2}$ and SKIP. ${ }^{3}$ This may be related to the differences of stroke aetiology across the trials with varying proportions of undetermined causes and intracranial atherosclerosis. Moreover, whether intravenous tPA infusion was completed in the bridging groups of these three trials prior to thrombectomy began was not reported.

Regarding patient populations studied, the three trials largely excluded drip-and-ship patients. Although SKIP $^{3}$ allowed transfer, the interval between tPA and groin puncture in this trial was around $8 \mathrm{~min}$, and therefore, it is fair to assume that the vast majority of patients presented directly. In the real world, intravenous tPA use is particularly efficient in drip-and-ship patients. The successful recanalisation rate of tPA for LVOS is between $10 \%$ and $40 \%$ depending on vessel size and timing of assessment, ${ }^{89}$ and it completely averts the need for endovascular treatment.

Notably, a recent meta-analysis summarising data of dEVT and BT across 30 studies involving 7191 patients in the BT group and 4891 patients in the dEVT group supported the continuous use of BT for eligible patients. Specifically, compared with patients in the dEVT group, patients in the BT group showed significantly better proportion of functional independence (mRS 0-2) at 90 days (OR 1.43 (95\% CI 1.28 to 1.61)), had lower 90-day mortality (OR 0.67 (95\% CI 0.60 to 0.75$)$ ), and achieved higher rates of successful recanalisation (modified Thrombolysis in Cerebral Ischemia score 2b-3) (OR 1.23 (95\% CI 1.07 to 1.42$)) .{ }^{10}$ However, we must acknowledge several limitations associated with the meta-analysis of observational studies. Only 5 out of the 30 studies in the meta-analysis were restricted to intravenous thrombolysis eligible patients and only one study (DIRECT-MT) had a randomised design. Importantly, this meta-analysis included a different patient population than the recent RCTs. In contrast to the three recent RCTs, transferred patients (a population where equipoise about BT clearly does not exist) were included in this meta-analysis. The meta-analysis also included wake-up and uncertain onset time strokes as well as posterior circulation occlusions. There were also remarkable differences in stroke severity with a median National Institutes of Health Stroke Scale score of only 10 in the meta-analysis vs $16-19$ in the recent RCTs. The median Alberta Stroke Program Early CT Score was unexpectedly lower in the metaanalysis at 6 versus in the RCTs at 7-9 despite the higher stroke severity in the latter. Therefore, meta-analysis of the actual RCTs of dEVT and BT will be much more informative.

\section{FUTURE DIRECTIONS}

While the current results do not necessarily support the dismissal of intravenous tPA in BT, they open up the opportunity for greater individualisation in the decisionmaking for patient who had LVOS presenting directly to thrombectomy-capable centres that can promptly perform endovascular treatment. ${ }^{5}$ In this context, BT may still be advantageous in a large proportion of patients but it might be reasonable to withhold tPA in certain situations such as those occlusions associated with very high clot burden (such as internal carotid artery occlusions), those with large baseline infarcts who may be at higher risk for intracerebral haemorrhage and those requiring stenting due to the need for dual antiplatelet agents. In addition, forgoing intravenous tPA reduces cost, which in many medical systems represents an important part of the decision-making process. Finally, it is critical to acknowledge the potential advantages of tenecteplase over tPA including easier and faster administration as well as higher rates of recanalisation in patients who had LVOS who are subsequently treated with thrombectomy. ${ }^{8}$ Therefore, in the future, additional exploration of BT remains valuable, especially with tenecteplase.

All patients were from Asia, which may not be generalised to other ethnic profiles. Additional ongoing RCTs comparing dEVT with BT including DIRECT-SAFE (A Randomized Controlled Trial of DIRECT Endovascular Clot Retrieval vs Standard Bridging Thrombolysis With Endovascular Clot Retrieval Within 4.5 Hours of Stroke Onset) in Australia and China (NCT03494920), SWIFT DIRECT (Solitaire With the Intention for Thrombectomy Plus Intravenous t-PA vs DIRECT Solitaire StentRetriever Thrombectomy in Acute Anterior Circulation Stroke) in Europe and Canada (NCT03192332), MR CLEAN-NO IV in the Netherlands (Multicenter Randomized Clinical Trial of Endovascular Treatment for Acute Ischemic Stroke in the Netherlands-No-Intravenous tPA; ISRCTN80619088) will hopefully provide more robust evidence.

Contributors $Y X$ and YP contributed equally. Manuscript draft and editorial design - YX and YP. Statistical analysis-YP. Critical revision of the manuscriptRGN, TGJ and ZR. Editorial design-YW.

Funding The authors have not declared a specific grant for this research from any funding agency in the public, commercial or not-for-profit sectors.

Competing interests None declared.

Patient consent for publication Not required.

Provenance and peer review Not commissioned; internally peer reviewed.

Open access This is an open access article distributed in accordance with the Creative Commons Attribution Non Commercial (CC BY-NC 4.0) license, which permits others to distribute, remix, adapt, build upon this work noncommercially, and license their derivative works on different terms, provided the original work is properly cited, appropriate credit is given, any changes made indicated, and the use is non-commercial. See: http://creativecommons.org/ licenses/by-nc/4.0/.

\section{ORCID iDs}

Yunyun Xiong http://orcid.org/0000-0003-1353-2295

Yuesong Pan http://orcid.org/0000-0003-3082-6789

Zeguang Ren http://orcid.org/0000-0002-7431-2643

Yongjun Wang http://orcid.org/0000-0002-9976-2341 


\section{REFERENCES}

1 Yang P, Zhang Y, Zhang L, et al. Endovascular thrombectomy with or without intravenous alteplase in acute stroke. N Engl J Med 2020;382:1981-93.

2 Zi W, Qiu Z, Li F, et al. Effect of endovascular treatment alone vs intravenous alteplase plus endovascular treatment on functional independence in patients with acute ischemic stroke: the devt randomized clinical trial. JAMA 2021;325:234-43.

3 Suzuki K, Matsumaru Y, Takeuchi M, et al. Effect of mechanical thrombectomy without vs with intravenous thrombolysis on functional outcome among patients with acute ischemic stroke: the SKIP randomized clinical trial. JAMA 2021;325:244-53.

4 Anderson CS, Robinson T, Lindley RI, et al. Low-dose versus standard-dose intravenous alteplase in acute ischemic stroke. $N$ Engl J Med 2016;374:2313-23.

5 Nogueira RG, Tsivgoulis G. Large vessel occlusion strokes after the direct-mt and SKIP trials: is the alteplase syringe half empty or half full? Stroke 2020;51:3182-6.
6 Lin C-J, Saver JL. Noninferiority margins in trials of thrombectomy devices for acute ischemic stroke: is the bar being set too low? Stroke 2019;50:3519-26.

7 Goyal M, Menon BK, van Zwam WH, et al. Endovascular thrombectomy after large-vessel ischaemic stroke: a meta-analysis of individual patient data from five randomised trials. Lancet 2016;387:1723-31

8 Campbell BCV, Mitchell PJ, Churilov L, et al. Tenecteplase versus alteplase before thrombectomy for ischemic stroke. $N$ Engl J Med 2018;378:1573-82.

9 Serna Candel C, Aguilar Pérez M, Hellstern V, et al. Recanalization of emergent large intracranial vessel occlusion through intravenous thrombolysis: frequency, clinical outcome, and reperfusion pattern. Cerebrovasc Dis 2019;48:115-23.

10 Wang Y, Wu X, Zhu C, et al. Bridging thrombolysis achieved better outcomes than direct thrombectomy after large vessel occlusion: an updated meta-analysis. Stroke 2021;52:356-65. 